\title{
FORMATION OF NUCLEAR SPIRALS IN BARRED GALAXIES
}

\author{
H.B. ANN AND PARIJAT THAKUR \\ Division of Science Education, Pusan National University, Busan 609-735, Korea \\ hbann@pusan.ac.kr, pthakur@pusan.ac.kr
}

\begin{abstract}
We have performed smoothed particle hydrodynamics ( $\mathrm{SPH}$ ) simulations for the response of the gaseous disk to the imposed moderately strong nonaxisymmetric potentials. The model galaxies are composed of the three stellar components (disk, bulge and bar) and two dark ones (supermassive black hole and halo) whose gravitational potentials are assumed to be invariant in time in the frame corotating with the bar. We found that the torques alone generated by the moderately strong bar that gives the maximum of tangential-to-radial force ratio as $\left(F_{T a n} / F_{\text {Rad }}\right)_{\max }=0.3$ are not sufficient to drive the gas particles close to the center due to the barrier imposed by the inner Lindblad resonances (ILRs). In order to transport the gas particles towards the nucleus $(r<100 \mathrm{pc})$, a central supermassive black hole $(\mathrm{SMBH})$ and high sound speed of the gas are required to be present. The former is required to remove the inner inner Lindblad resonance (IILR) that prevents gas inflow close to the nucleus, while the latter provides favourable conditions for the gas particles to lose their angular momentum and to spiral in. Our models that have no IILR show the trailing nuclear spirals whose innermost parts reach close to the center in a curling way when the gas sound speed is $c_{s} \gtrsim 15 \mathrm{~km} \mathrm{~s}^{-1}$. They resemble the symmetric two-armed nuclear spirals observed in the central kiloparsec of spiral galaxies. We found that the symmetric two-armed nuclear spirals are formed by the hydrodynamic spiral shocks caused by the gravitational torque of the bar in the presence of a central SMBH that can remove IILR when the sound speed of gas is high enough to drive a large amount of gas inflow deep inside the ILR. However, the detailed morphology of nuclear spirals depends on the sound speed of gas.
\end{abstract}

Subject headings: galaxies: evolution — galaxies: nuclei — galaxies: spiral — methods: numerical — galaxies: kinematics and dynamics - galaxies: structure 


\section{INTRODUCTION}

Recent observations by ground-based telescopes with adaptive optics and Hubble Space Telescope (HST) showed a variety of features such as nuclear spirals, nuclear rings, and nuclear bars in the centers of galaxies. Among them, nuclear spirals are most frequently observed ones in active galaxies (Regan \& Mulchaey 1999; Martini \& Pogge 1999; Pogge \& Martini 2002) as well as in normal spirals galaxies (Phillips et al. 1996; Laine et al. 1999, 2001). The existence of these nuclear features had been suggested by the ground-based observation of the blue nuclei or peculiar nuclear structures since 1950s (Haro 1956; Sersic 1958), but analysis of their detailed morphology was impossible due to lack of resolution. However, thanks to the high resolution capability of $H S T$, it is now possible to resolve the nuclear features down to scales of $10 \mathrm{pc}$ or less for the galaxies within $\sim 20 \mathrm{Mpc}$.

Just like the spiral arms in the disk of spiral galaxies, nuclear spirals have a diversity of morphology such as symmetric two-armed spirals, one-armed spirals, and chaotic ones (Martini et al. 2003). Because of the diverse morphology, the formation mechanism of nuclear spirals is difficult to be understood. However, some mechanisms have been proposed to explain the formation of chaotic or "flocculent" and symmetric two-armed or "granddesign" nuclear spirals. The flocculent nuclear spirals are suggested to be formed by the acoustic instability (Elmegreen et al. 1998; Montenegro et al. 1999), whereas the granddesign nuclear spirals are shown to be generated by the gas density waves supported by the pressure forces in the non-self-gravitating gaseous disks (Englmaier \& Shlosman 2000) or by the hydrodynamic shocks induced by the non-axisymmetric potential of the large scale bar (Maciejewski et al. 2002). The weak nuclear spirals whose wave amplitude is considered to be linear can be explained by the density waves since the gas density waves can be applied to the regions where no shocks exist, while the strong nuclear spirals are thought to be the hydrodynamic shocks because their amplitude is likely to be out of the linear regime (Maciejewski et al. 2002).

Since the morphology of nuclear spirals depends on the sound speed of gas and the shape of potential in the nuclear regions of galaxies (Englmaier \& Shlosman 2000), it is important to understand their effect on the gas flows inside the ILR. The effect of sound speed on the gas flows in hydrodynamical simulations is quite well known (Englmaier \& Gerhard 1997; Patsis \& Athanassoula 2000; Maciejewski et al. 2002). However, the previous studies except for that by Maciejewski et al. (2002) mainly concerned with the gas flows outside the nuclear regions of galaxies. Using SPH method, Englmaier \& Gerhard (1997) and Patsis \& Athanassoula (2000) showed that off-axis shock developes at low sound speed, while on-axis one at high sound speed. But the resolutions of their simulations are not high enough to analyze the nuclear morphology in detail. 
The physical parameter that determines the shape of the gravitational potential in the nuclear regions of galaxies is the central mass concentration which can be much affected by the presence of an SMBH even though the mass of an SMBH is less than $1 \%$ of the total mass of a galaxy. Since nuclear spirals are found to be preponderant in active galaxies (Martini et al. 2003) where SMBHs are expected to be present, it seems to be important to understand the effect of the central SMBH as well as that of the sound speed on the gas flows inside the ILR. Thus, the purpose of the present paper is to see the behaviour of the gas flows in the presence of the central SMBHs, which leads to the formation of nuclear spirals. For this purpose, we employ the SPH technique to solve the hydrodynamical equations for the gas flow in the model galaxies with central SMBHs. We have also included the self-gravity of the gas in our calculations, since this can play significant role in the high density regions like shocks.

The remainder of this paper is organised as follows. In $\S 2$ we describe the hydrodynamic modeling of the gas flows. In $\S 3$ we present the results of our simulations and the discussion of our results is given in $\S 4$. The conclusions are presented in $\S 5$.

\section{HYDRODYNAMIC MODELING OF GAS FLOWS}

\subsection{Numerical Method}

We have used PMSPH method for modelling the gas flows in barred galaxies. The hydrodynamical equations for the gas flow was solved by the smoothed particle hydrodynamics (SPH) method, while the self-gravity of gas was calculated by particle-mesh (PM) N-body technique. In the SPH, fluid elements constituting the system are sampled and represented by particles, and the dynamical equations are described by the Lagrangian form of the hydrodynamic conservation laws. Due to its Lagrangian nature, there is no grid to impose any artificial restrictions on the global geometry of the systems under study or any mesh-related limitations on the dynamic range in spatial resolution. As a result, the high numerical resolution is naturally achieved in high density regions like shocks (Fukuda et al. 1998), which is necessary in our present problem of understanding the response of gaseous disk in the nuclear regions of galaxies. This is the reason why we have used the PMSPH code (Fux 1999, 2001) which Roger Fux kindly made available. We describe here briefly the necessary specifications relevant to the particular aspects of our code and will refer the readers to Fux (1999, 2001) for the detailed description of our code and to Monaghan (1992) for general properties of solving the Euler's equation of motion using popular Lagrangian method. 


\subsubsection{Gas Dynamics}

The relevant form of the Euler equation of motions can be written as

$$
\frac{d \mathbf{v}}{d t} \equiv \frac{\partial \mathbf{v}}{\partial t}+(\mathbf{v} \cdot \nabla) \mathbf{v}=-\frac{\nabla(P+\Pi)}{\rho}-\nabla \Phi,
$$

where $\mathbf{v}(\mathbf{r})$ is the velocity field, and $\rho(\mathbf{r}), P(\mathbf{r})$ and $\Pi(\mathbf{r})$ are the density, pressure and artificial viscosity of the gas, respectively. $-\nabla \Phi$ is the gravitational force, where $\Phi$ includes potentials of the gas as well as the stellar and dark components.

In the $\mathrm{SPH}$, the density is evaluated directly at each particle position $\mathbf{r}_{i}$ in space by summing the contributions from the density profiles of neighbouring particles and is represented by

$$
\rho_{i} \equiv \rho\left(\mathbf{r}_{i}\right)=\sum_{j=1}^{N_{g}} m_{j} W\left(\mathbf{r}_{i}-\mathbf{r}_{j}, h\right),
$$

where $N_{g}$ is the number of gas particles, $m_{j}$ is the mass of individual particles, $W(\mathbf{r}, h)$ is the kernel function and $h$ is the smoothing length which defines the local spatial resolution and is proportional to the local mean interparticle spacing. Here in our code the adopted SPH kernel is a spherically symmetric spline that vanishes outside $2 h$ because of its finite spatial extension. Thus, the particles only within a radius of $2 h$ will contribute to the smoothed estimates for this kernel.

Since we assume an isothermal gas, the equation of state is expressed as

$$
P_{i}=\frac{c_{s}^{2}}{\gamma} \rho_{i},
$$

with the adiabatic index $\gamma=1$ and $c_{s}$ as an effective sound speed which can be interpreted globally as the velocity dispersion of the interstellar clouds. The internal energy of the gas is at any time and everywhere constant, and in particular, there is no energy equation involved. The homogeneous velocity dispersion of warm gas component in the external galaxies (e.g., van der Kruit \& Shostak 1984) partly justifies this assumption. It means that cooling and heating exactly cancel each other and the energy released by shocks is instantaneously radiated away. With the isothermal assumption of the gas, the pressure gradient term in the Euler's equation simply takes the form

$$
\left(\frac{\nabla P}{\rho}\right)_{i} \approx \frac{c_{s}^{2}}{\gamma} \sum_{j=1}^{N_{g}} m_{j}\left(\frac{1}{\rho_{i}}+\frac{1}{\rho_{j}}\right) \nabla_{i} W\left(\mathbf{r}_{i}-\mathbf{r}_{j}, h\right), \text { where } \gamma=1 .
$$

For accurate treatment of flow near shocks, it is necessary to introduce the artificial viscosity. In our code, the artificial viscosity is calculated as in Benz (1990) with Balsara 
(1995)'s correction, i.e.,

$$
\left(\frac{\nabla \Pi}{\rho}\right)_{i} \approx \sum_{j=1}^{N_{g}} m_{j} \widetilde{\Pi_{i j}} \nabla_{i} W\left(\mathbf{r}_{i}-\mathbf{r}_{j}, h\right)
$$

where

$$
\begin{gathered}
\widetilde{\Pi_{i j}} \equiv \frac{\Pi_{i j}}{\rho^{2}}= \begin{cases}f_{i j} \frac{\left(-\alpha \mu_{i j} c_{s}+\beta \mu_{i j}^{2}\right)}{\rho_{i j}} & \text { if }\left(\mathbf{v}_{i}-\mathbf{v}_{j}\right) \cdot\left(\mathbf{r}_{i}-\mathbf{r}_{j}\right) \leq 0, \\
0 & \text { otherwise }\end{cases} \\
\mu_{i j}=\frac{h\left(\mathbf{v}_{i}-\mathbf{v}_{j}\right) \cdot\left(\mathbf{r}_{i}-\mathbf{r}_{j}\right)}{\left(\mathbf{r}_{i}-\mathbf{r}_{j}\right)^{2}+\xi h^{2}},
\end{gathered}
$$

with $\alpha=1.0, \beta=2.5, \xi=0.01$ and $\rho_{i j}=\left(\rho_{i}+\rho_{j}\right) / 2$. Here, $f_{i j}=\left(f_{i}+f_{j}\right) / 2$ is the correction factor which was introduced by Balsara (1995) to avoid the energy dissipation in pure shearing flows and has the following form:

$$
f_{i}=\frac{\left|\langle\nabla \cdot \mathbf{v}\rangle_{i}\right|}{\left|\langle\nabla \cdot \mathbf{v}\rangle_{i}\right|+\left|\langle\nabla \times \mathbf{v}\rangle_{i}\right|+0.0001 c_{s} / h}
$$

Furthermore, in above equation (6), the term with the coefficient $\alpha$ corresponds to the bulk viscosity, whereas the term with the coefficient $\beta$ represents to the von Neumann-Richtmyer viscosity. The von Neumann-Richtmyer viscosity is necessary to handle strong shocks. On the other hand, the bulk viscosity is required to damp post-shock oscillations.

In order to achieve high-resolution, it is necessary to treat smoothing length $h$ properly since it determines local spatial resolution. Basically, the SPH assumes that $h$ is the same for all the particles, which would give relatively more accurate estimates in the high density regions than in the lower ones. Thus, it is desirable to compute smoothed quantities with the same level of accuracy everywhere in order to achieve both consistency and efficiency in the smoothed estimates. For this purpose, our code considers spatially variable smoothing length $h$ to assign an individual smoothing length $h_{i}$ to each particle in such a manner that the number of neighbour particles $N_{i}$ to each particle always remains as close as possible to a fixed number $N_{o}=35$. This allows us to save computing time significantly while keeping spatial resolutions high enough to resolve the shocks (Fux 1999, 2001). In this scheme, two particles $i$ and $j$ are defined as mutual neighbours if $i \neq j$ and $\left|\mathbf{r}_{i}-\mathbf{r}_{j}\right|<2 h_{i j}$, where $h_{i j}=\left(h_{i}+h_{j}\right) / 2$ is the symmetrized smoothing length which is supplied in all the above formulae for pressure gradient and artificial viscosity to ensure momentum conservation as well as to treat shock phenomena in a gaseous disk correctly. In our code, the update of the smoothing lengths was made at each time step according to a general three-dimensional scaling law:

$$
\frac{h_{i}}{h_{o}}=\left(\frac{N_{i}+1}{N_{o}+1} \frac{\rho_{o}}{\rho_{i}}\right)^{1 / 3}
$$


where $h_{o}$ and $\rho_{o}$ are constants, $N_{i}$ is number of neighbours of particle $i$, and +1 is added to take into account particle $i$. Since the determination of the density $\rho_{i}$ at the particle location requires the $h_{i}$ 's which are not known a priori, it is better to take the time derivative of equation (9) and substitute the continuity equation to yield

$$
\dot{h_{i}} \equiv \frac{d h_{i}}{d t}=\frac{1}{3} h_{i}\left(\frac{1}{N_{i}+1} \frac{d N_{i}}{d t}+[\nabla \cdot \mathbf{v}]_{i}\right) .
$$

In the traditional approach of Benz (1990), the term $d N_{i} / d t$ was set to zero to ensure a constant number of neighbours. However, this does not prevent a slow numerical departure of the $N_{i}$ 's from $N_{o}$ with time. Instead, our code takes the advantage of the $N_{i}$ term to damp such departures by setting

$$
\frac{d N_{i}}{d t}=\frac{N_{i}-N_{o}}{\eta \triangle t}
$$

in equation (10) and integrates the resulting equation along with the equations of motion. The parameter $\eta$ is used to control the damping rate per time step $\Delta t$ and should be significantly greater than 1 to avoid sharp changes in non-gravitational forces. We have fixed $\eta=5$ in all our simulations.

Since the computation time depends much on the smoothing lengths of individual particles, it is customary to impose quite a large lower limit on the minimum smoothing length $h_{\text {min }}$ to save computing time. However, we have used $h_{\text {min }}=0.1 \mathrm{pc}$ to resolve structures down to pc scale. Our adopted $h_{\text {min }}$ is an order of magnitude smaller than the $h_{\text {min }}$ 's employed in the previous works (Heller \& Shlosman 1994; Fukuda et al. 2000). Imposing virtually no limit on the $h_{\text {min }}$ was possible due to the fast neighbour searching algorithm adopted in our code. However, the smallest smoothing length actually achieved in our simulations is $\sim 2$ pc which is still smaller than the $h_{\text {min }}=5$ pc used in the high-resolution simulations of Fukuda et al. (2000). Another important aspect of our code which is worth to be noted here is the adoption of a synchronised version of the standard leap-frog time integrator (e.g., Hut et al. 1995). This integrator is known to well conserve the total energy and the total angular momentum and also incorporates an adaptative time-step to temporally resolve the high density shocks in the gaseous disk (see Fux 1999, 2001).

\subsubsection{Self-gravity of Gas}

Since the mass of the gaseous disk is assumed to be only a tiny fraction of the model galaxy $(\sim 1 \%)$, our gaseous disk is not a self-gravitating one. But, we have included the self-gravity of gas in our calculations because this can play significant role in the high density regions such as shocks. In particular, the effect of gas self-gravity can not be ignored in the 
later evolution of gaseous disk, especially in the nuclear disk where the nuclear spirals are supposed to be formed. For computing the self-gravity of gas, we have used particle-mesh (PM) technique with polar-cylindrical grid geometry and variable homogeneous ellipsoidal kernel for the softening of the short range forces (Pfenniger \& Friedli 1993). Since our simulations are confined in two-dimensions to achieve a high spatial resolution, we have used a single grid version of PMSPH code of Fux $(1999,2001)$. For more detailed description of the PM technique, we simply refer the readers to Fux $(1999,2001)$ which itself follows Pfenniger \& Friedli (1993) for this technique. Owing to the intrinsic nature of polar-cylindrical grid, the radial and azimuthal resolutions are increased toward the center where a variety of features are likely to be developed due to the gas inflow driven by the bar.

\subsection{The Model Galaxy}

We assume that a model galaxy is composed of the three stellar components (bulge, disk and bar) and two dark components (SMBH and halo). The properties (density, size and structure) of all these potential generating components are assumed to be invariant in time in the frame corotating with the bar. We employ the same analytic potentials as those of Ann \& Lee (2000) and Ann (2001) for the stellar components and the halo.

\subsubsection{The Potential}

The halo component, which gives rise to the flat rotation curve at outer radii, is assumed to have a logarithmic potential with finite core radius,

$$
\Phi(r)_{h a l o}=\frac{1}{2} v_{0}^{2} \ln \left(R_{h}^{2}+r^{2}\right)+\text { const }
$$

where $R_{h}$ is the halo core radius, and $v_{0}$ is the constant rotation velocity at large $r$. For the disk component, we adopt the Freeman's (1970) exponential disk which has the following form for the potential,

$$
\begin{aligned}
\Phi(r)_{d i s k} & =\pi G \Sigma_{0} r\left[I_{0}\left(\frac{r}{2 R_{d}}\right) K_{1}\left(\frac{r}{2 R_{d}}\right)\right. \\
& \left.-I_{1}\left(\frac{r}{2 R_{d}}\right) K_{0}\left(\frac{r}{2 R_{d}}\right)\right] .
\end{aligned}
$$

Here $R_{d}$ is the disk scale length, $\Sigma_{0}$ is the central surface density, and $I_{0}, K_{0}, I_{1}$, and $K_{1}$ are the modified Bessel functions. The total disk mass is simply $M_{d i s k}=2 \pi \Sigma_{0} R_{d}^{2}$. As for 
the bulge component, we have assumed the Plummer model (Binney \& Tremaine 1987)

$$
\Phi(r)_{b u l g e}=-\frac{G M_{\text {bulge }}}{\sqrt{r^{2}+R_{b}^{2}}},
$$

where $R_{b}$ is a parameter which controls the size of bulge. The bulges of real galaxies can be better represented by de Vaucouleurs' $R^{1 / 4}$-law, but we have taken Plummer model for simplicity.

The bar is a triaxial component in three-dimension. However, since our simulation is restricted to the two-dimensional disk, we have adopted the following form of gravitational potential proposed by Long \& Murali (1992) for the bar component,

$$
\Phi(r)_{b a r}=\frac{G M_{b a r}}{2 a} \log \left(\frac{x-a+T_{-}}{x+a+T_{+}}\right),
$$

where $T_{ \pm}=\sqrt{\left[(a \pm x)^{2}+y^{2}+b^{2}\right]}$. Here $a$ and $b$ are the needle length and softening length, respectively. Because Long \& Murali (1992)'s potential is of a softened needle, it does not describe an axisymmetric system for $a=b$. The parameter $a / b$ defines the elongation of the bar which is proportional to the axis ratio $\tilde{a} / \tilde{b}$ of Freeman's (1966) flattened homogeneous ellipsoid bar when $a / b \geq 2$ (see Fig. 3 of Long \& Murali 1992), where $\tilde{a}$ and $\tilde{b}$ are the major and minor axes of flattened homogeneous ellipsoid bar. After readjusting the relations established in Long \& Murali (1992) for the parameters of these two bars, we found that $\tilde{a} / \tilde{b}=1.05 a / b$ with $\tilde{a}=1.15 a$ and $\tilde{b}=1.1 b$.

We assume the point mass potential for the central SMBH component,

$$
\Phi(r)_{S M B H}=-\frac{G M_{S M B H}}{\sqrt{r^{2}+\epsilon_{S M B H}^{2}}},
$$

where $M_{S M B H}$ is the mass of the central SMBH and $\epsilon_{S M B H}$ is a softening parameter.

\subsubsection{Set up of the Gaseous Disk}

We assumed an isothermal uniform gaseous disk with an infinitesimal thickness. Since our aim is to simulate the response of the gaseous disk in early type galaxies, the mass of gaseous disk was chosen to be $1 \%$ of the total visible mass of the model galaxy. To make an initial uniform gaseous disk, we distributed $2 \times 10^{4} \mathrm{SPH}$ particles randomly within the radius of $5 \mathrm{kpc}$ with the initial rotational velocities balancing to the centrifugal acceleration caused by the axisymmetric components. The initial resolution length, defined by $\sqrt{m_{i} / 4 \pi \Sigma\left(R_{g}\right)}$, was found to be $\sim 20 \mathrm{pc}$, where $m_{i}$ and $\Sigma\left(R_{g}\right)$ are the mass of each gas particle (in the unit 
of $\left.M_{s c}=2 \times 10^{11} \mathrm{M}_{\odot}\right)$ and the surface density of the initial gaseous disk of radius $R_{g}=5$ $\mathrm{kpc}$ (in the unit of $\Sigma_{s c}=2 \times 10^{5} \mathrm{M}_{\odot} \mathrm{pc}^{-2}$ ), respectively. The evolution of gaseous disk is turned on when we introduce the non-axisymmetric potential of the bar. But the strength of bar is increased gradually within a half bar rotation period to avoid spurious response of the gaseous disk, where bar rotation period $\tau_{\text {bar }}$ is $\sim 1.4 \times 10^{8} \mathrm{yr}$.

\subsubsection{Model Parameters}


TABLE 1

MODEL PARAMETERS

\begin{tabular}{ccc}
\hline \hline Model & $c_{s}\left[\mathrm{~km} \mathrm{~s}^{-1}\right]$ & $M_{S M B H}$ \\
\hline M1 & 10. & 0.0 \\
M2 & 10. & 0.002 \\
M3 & 15. & 0.002 \\
M4 & 20. & 0.002 \\
\hline
\end{tabular}


We have considered four models M1-M4 which have the same mass models except for the presence of the central SMBH but different hydrodynamic properties. Basically the mass distributions of the model galaxies are assumed to be similar to that of an early type barred galaxy $(\sim \mathrm{SBa})$ of which disk-to-bulge mass ratio $(\mathrm{D} / \mathrm{B})$ is 2 and the fractional mass of the bar $M_{b a r} / M_{G}$ is 0.2 . In order to reproduce the typical rotation curves of early type barred galaxies, the scale lengths of bulge, disk, bar and halo are chosen to be $R_{b}=0.5 \mathrm{kpc}$, $R_{d}=3.0 \mathrm{kpc}, a=3.0 \mathrm{kpc}$ and $R_{h}=15.0 \mathrm{kpc}$, respectively. Since the total visible mass of the model galaxies $M_{G}$ is assumed to be $\sim 4 \times 10^{10} \mathrm{M}_{\odot}$, the masses of the gas, bulge, disk and bar components in the unit of $M_{s c}=2 \times 10^{11} \mathrm{M}_{\odot}$ are $M_{\text {gas }}=0.002, M_{\text {bulge }}=0.054$, $M_{\text {disk }}=0.104$ and $M_{b a r}=0.04$, respectively. Furthermore, the rotation velocity of halo and the SMBH softening parameter are fixed at $v_{0}=186 \mathrm{~km} \mathrm{~s}^{-1}$ and $\epsilon_{S M B H}=1 \mathrm{pc}$, respectively. We chose moderately strong bars that gives the maximum of tangential-to-radial force ratio as $\left(F_{\text {Tan }} / F_{\text {Rad }}\right)_{\max }=0.3$ by assuming $a / b=3 a / b=3$ with $M_{b a r} / M_{G}=0.2$ for all the models. In the models with a central SMBH, we assumed the mass of an SMBH $\left(M_{S M B H}\right)$ to be $\sim 1 \%$ of the total visible mass of host galaxy to remove the IILR effectively. The fractional mass of the SMBH assumed in the present models is somewhat larger than the observed one (e.g., Marconi \& Hunt 2003). But, we take it for an easy comparison with the previous works (e.g., Fukuda et al. 2000). Since the primary role of the SMBH in our models is to remove the IILR and there is not much difference in the gas flows in models with different SMBHs unless they are too small to remove the IILR (Ann \& Lee 2004), it should give us insight into the inner dynamics of galaxies that might host SMBHs. We list all the models in Table 1 where $c_{s}$ and $M_{S M B H}$ stand for the effective sound speed of the gas and the mass of $\mathrm{SMBH}$ in the unit of $M_{s c}=2 \times 10^{11} \mathrm{M}_{\odot}$, respectively.

As clearly seen in Table 1, the model M1 does not have a central SMBH component, while the other models contain SMBHs at the center. Hence, the model M1 was selected here to compare the effect of SMBH on the gas flow, whereas the models M2-M4 were chosen to see the effect of sound speed in the presence of an SMBH. According to the terminology of Maciejewski (2003), the models M1-M2 that assume $c_{s}=10 \mathrm{~km} \mathrm{~s}^{-1}$ are the cold gas models and remaining two models (i.e., the models M3-M4) can be treated as the hot gas models. Since the models M2-M4 have the same mass distribution, we present the rotation curves and angular frequencies curves of M1 and M2 in Fig. 1. The top left panel of Fig. 1 represents the rotational velocities of the bulge, disk, bar and halo components of the model M1 as a function of radius, while top right panel shows rotational curves of the model M2 which includes the contribution of the central SMBH besides all other components those in the model M1. Due to non-axisymmetric nature of the bar, its contribution to the rotational velocity is included here after averaging it axisymmetrically (Hasan et al. 1993). As can be seen clearly in the top panels of Fig. 1, the presence of SMBH does not much affect 
the rotational velocities except for the very vicinity of galactic nuclei. However, as evident from the bottom panels of Fig. 1 where we present the angular frequencies corresponding to the rotational velocity generated by the axisymmetric components as a function of radius, the existence of the IILR critically depends on the presence of SMBH. For the adopted bar pattern speed of $\Omega_{p}=44.6 \mathrm{~km} \mathrm{~s}^{-1} \mathrm{kpc}^{-1}$, which makes the corotation radius $\left(R_{C R}\right)$ similar to $1.2 a$, we have two ILRs (i.e., the IILR and the outer inner Lindblad resonance (OILR)) in the model M1 but no IILR in the model M2. The locations of IILR and OILR are, respectively, found at $0.3 \mathrm{kpc}$ and $1.5 \mathrm{kpc}$ in the model M1, whereas the location of the single ILR in the model M2 is the same as that of the OILR in the model M1. 
TABLE 2.

AVERAGE PITCH ANGLE FOR NUCLEAR SPIRALS

\begin{tabular}{ccccc}
\hline \hline Model & $c_{s}\left[\mathrm{~km} \mathrm{~s}^{-1}\right]$ & \multicolumn{3}{c}{$\overline{i_{p}}[$ degree $]$} \\
& & $5 \tau_{\text {bar }}$ & $10 \tau_{\text {bar }}$ & $20 \tau_{\text {bar }}$ \\
\hline M2 & 10 & 12.8 & 10.5 & 9.6 \\
M3 & 15 & 13.2 & 12.5 & 11.8 \\
M4 & 20 & 14.5 & 13.4 & 12.5 \\
\hline
\end{tabular}




\section{RESULTS}

\subsection{Time Evolution of the Gaseous Disk}

\subsubsection{Models with $S M B H$}

Since the global morphological evolutions of the gaseous disk of our models are similar, we present this only for the model M3 which considers the medium value of the sound speed (i.e., $c_{s}=15 \mathrm{~km} \mathrm{~s}^{-1}$ ) employed in the present models. However, the evolution of the nuclear regions are presented for all the models to understand the behaviour of the gas flow in the central kilo-parsecs of barred galaxies. Here our results are shown on the frame co-rotating with the bar which always lies horizontally. Fig. 2 shows the global distribution of the gas particles in the model M3 at the four evolution times that are given in the upper left corner of each panel. At time of $0.75 \tau_{\text {bar }}$, the gaseous disk is distorted in the direction leading the bar major axis by $\sim 135^{\circ}$, displaying the spiral shocks such as the spiral shocks in the outer disk, 4/1-spiral shocks just inside the bar radius (Englmaier \& Gerhard 1997), and the principal shocks along the leading edges of the bar which are almost straight, off-centered and inclined to the bar major axis. These shocks are generic features for various bar potentials and are independent of sound speed in the gas (Maciejewski et al. 2002). Moreover, they are made by the gas flows driven by the gravitational torques of the bar and reflect the orbital families in the underlying potential. As shown in the velocity field of the model M3 at the time of $0.75 \tau_{\text {bar }}$ in Fig. 3, the gas particles move inward alongside the principal shocks following the x1-orbits and then swing around near the ILR towards the far side of the symmetric shocks. Some of the particles move to the x2-orbits inside the ILR and others move outward reaching the far side-shock again. The winding inner part of the principal shocks inside the ILR are made by this process of orbital switching from the x1-orbits which align with the bar axis to the x2-orbits perpendicular to the bar (Athanassoula 1992; Wada 1994; Knapen et al. 1995; Englmaier \& Shlosman 2000).

At the time of $5 \tau_{\text {bar }}$, the three above mentioned spiral shocks become less prominent, but we can see the trailing spiral arms in the outer disk. The nuclear region inside the ILR looks like oval disk with a hole in the center which seems to lead the bar by more than $45^{\circ}$. The reason for the weakening of the shock features are due to the reduced gas flows arising from the lack of gas recycling in our models. In the later evolution times, shocks become very weak, since most of the particles inside the bar radius have already moved into the central kiloparsec. This makes the completely filled nuclear disk. Furthermore, it also seems that the outer spiral arms get disappeared and evolve to more symmetric structure. Since we do not employ gas recycling, our models do not reach to the steady state even at the time of $\sim 20 \tau_{\text {bar }}$. However, the global morphology of the gaseous disk does not change much and 
it reaches to a quasi-steady state after $\sim 20 \tau_{\text {bar }}$.

Fig. 4 shows the evolution of gas distribution in the central kiloparsecs of the model M3. Now we can clearly see a two-armed spiral in the nuclear disk. The early phase of evolution (i.e., at $0.75 \tau_{\text {bar }}$ ), shown in the top left panel of Fig. 4, is characterized by a development of open symmetric spiral patterns that are connected to the principal shocks in the leading edge of the bar. These spiral patterns display clumpy substructures that are similar to the ripples and clumps observed in the recent numerical simulations for spiral shocks (Wada \& Koda 2004) which suggest that these substructures are made by the wiggle instability. However, this clumpy structure disappears shortly within $\sim \tau_{b a r}$ and a smooth two-armed nuclear spiral pattern begins to emerge.

At the time of $5 \tau_{\text {bar }}$, the trailing nuclear spiral arms are well developed and they extend more than $\frac{3}{2} \pi$ in the azimuthal direction but the density of the spiral arms decreases significantly after $\frac{3}{2} \pi$. In the later evolution times, the nuclear spiral becomes more tightly wound and its innermost parts reach closer to the center in a curling manner. This results in the increase of winding angle of nuclear spiral which becomes greater than $3 \pi$ at the time of $20 \tau_{b a r}$. At this time, there are much more particles in the nuclear disk but the arm-interarm density contrast becomes smaller than earlier ones.

In order to see the effect of the sound speed on the gas flows in the nuclear regions of galaxies, we present the evolutions of the central kiloparsec of the models M2 and M4 in Fig. 5. Despites the similarity in the global morphological evolutions of the models M2 and M4, the morphological evolutions of the central kiloparsec of the two models, both of which show the formation of nuclear spirals, are quite different. Besides the morphological difference of nuclear spirals, the evolution of the model M4 that assumes $c_{s}=20 \mathrm{~km} \mathrm{~s}^{-1}$ is much faster than the model M2 whose sound speed is half of the model M4. As a result, nuclear spirals are formed in the earlier time for the models with higher sound speed than those with lower sound speed.

However, the earlier evolutions of the nuclear regions of the gaseous disks with different sound speeds are not much different. They are characterized by the open spiral patterns of clumpy substructures, as similar to those of the model M3. The drastic changes have been noticed at the later evolutions. At the time of $5 \tau_{\text {bar }}$, the model M2 shows rather tightly wound symmetric two-armed nuclear spiral whose winding angle is less than $\frac{3}{2} \pi$, while the model M4 shows spiral pattern similar to that of the model M3 but extends more closer to the center, making its winding angle greater than $2 \pi$. It is also apparent that the models with higher sound speeds have larger number of the gas particles in their nuclear disks. But the arm-interarm density contrast is highest in the models with the lowest sound speed. At this time, the density contrasts between arm and interarm regions are greater than those of 
the principal shocks due to the significant reduction of the gas flows along the bar.

At the time of $10 \tau_{\text {bar }}$, the nuclear disk of the model M4 shows a well developed symmetric two-armed nuclear spiral whose innermost parts reach very close to the center to wind it upto $\sim 3 \pi$, whereas that of the M2 shows a symmetric two-armed spiral whose density decreases significantly after $\sim \frac{3}{2} \pi$ in the azimuthal angle. In the later evolution time $\left(\sim 20 \tau_{\text {bar }}\right)$, the innermost parts of nuclear spiral arms of the model M4 are no longer resolved after $2 \pi$ due to the formation of clumps close to the center, while the nuclear spiral of the model M2 evolves to more tightly wound ring-like spiral whose winding angle is appeared to be more than $2 \pi$. Also, there seems to be little gas in the central hundred parsecs of the model M2. The diameter of the nuclear disk in quasi-steady state evolution also seems to depend on the sound speed of gas. The models with lower sound speeds allow larger nuclear disk than those with high sound speed.

The open spiral is defined by the larger pitch angle $i_{p}$, while relatively smaller pitch angle $i_{p}$ represents tightly wound one. Since the pitch angles $i_{p}$ of the above mentioned symmetric two-armed nuclear spirals do not vary much along the radius, we have measured the mean pitch angle $\overline{i_{p}}$ in order to define their openness. Table 2 summarizes the average pitch angles $\overline{i_{p}}$ of nuclear spirals at the three evolution times of $5 \tau_{\text {bar }}, 10 \tau_{\text {bar }}$ and $20 \tau_{\text {bar }}$ for the models M2-M4. As can be seen clearly in Table 2 , the average pitch angle $\overline{i_{p}}$ increases with the sound speed which suggests that the nuclear spiral arms open out as sound speed in the gas increases. Furthermore, it is also apparent that the symmetric two-armed nuclear spirals become slightly more tightly wound with time.

\subsubsection{A Model without an $S M B H$}

The evolution of the central kiloparsec of the model M1 is much different from the other models. However, the global morphological evolution of the model M1 is nearly similar to that of the model M2 which has identical initial conditions as those in the model M1 except for the presence of an SMBH. The drastic difference in the nuclear evolution is due to the presence of the IILR in the model M1 that can prevent the inflow of gas toward the nucleus. Fig. 6 shows the evolutions of the central kiloparsec of the model M1 at six evolution times which are indicated by the numbers in the upper left corner of each frame. The early evolution at $0.75 \tau_{\text {bar }}$ is similar to that of the model M2 but the snapshot at $5 \tau_{\text {bar }}$ already shows much difference. There is a development of a ring inside the two symmetric spiral arms near $\sim 1 \mathrm{kpc}$. This ring is located near the IILR and it becomes more elongated until $\sim 12 \tau_{\text {bar }}$, resulting in the development of the highly elongated oval-shaped nuclear ring with its orientation close to the bar minor axis. At the time of $20 \tau_{\text {bar }}$, the eccentricity of the 
oval-shaped nuclear ring decreases and some of the gas particles around the IILR acquire angular momentum to come out from this resonance due to the positive bar torque there (Combes et al. 2002). As a result, nearly circular nuclear ring-like structure with a large diffusion of the gas particle around its boundary is found.

The later evolutions (from $\sim 10 \tau_{\text {bar }}$ ) of the M1 model lead to the formation of a pair of leading nuclear spirals that emerge from the nuclear ring structure just outside the IILR. It reaches to the quasi-steady state at $\sim 25 \tau_{\text {bar }}$. There seems to be only a negligible number of the gas particles inside the IILR due to the change in the sense of the bar torque at the IILR (Combes et al. 2002). Similar morphologies were also observed in the simulations of Heller et al. (2001) who called the two-armed spiral and ring structure as a double rings before they merger into an elongated nuclear ring. But, the leading nuclear spirals are transient features in their models and the elongated nuclear ring persists for the end of run with varying orientation. The reason for this discrepancy in the persistence of the leading nuclear spirals is not clear. It might be due to the differences in the mass models as well as the neglection of gas self-gravity in their models. However, there seems to be not much difference in the potential shapes which directly affect the gas flows, since the evolution until about $\sim 10 \tau_{\text {bar }}$ is virtually identical. Thus, it seems quite plausible that the difference in the later evolution is due to the effect of gas self-gravity on the gas flow. In order to see whether the gas self-gravity is the real cause for the discrepancies between our models and their ones, we performed a simulation identical to the model M1 except for the neglection of gas self-gravity, which shows the transient leading nuclear spirals similar to those of Heller et al. (2001). Moreover, the evolution of the elongated nuclear ring after the formation of the transient leading nuclear spirals, which is characterized by the highly elongated shape and continuous change in the orientation, is also observed in this case. This suggests that the self-gravity of gas help to sustain the leading nuclear spirals by enhancing density contrast. However, the different treatment of hydrodynamical parameters such as artificial viscosity and limiting smoothing length $\left(h_{\min }\right)$, along with the different assumption of the mass models may also play some roles in making the discrepancies between our model M1 and those of Heller et al. (2001).

\subsection{Bar-driven Nuclear Spiral Shocks}

As described in the previous sections, all the models in the present study show a development of well defined nuclear spiral in their quasi-steady state evolution. Since these nuclear spirals seem to be connected with the principal shocks which are thought to be genuine hydrodynamical shocks (Athanassoula 1992; Englmaier \& Gerhard 1997; Maciejewski 
et al. 2002), it is quite plausible that, like the principal shocks, they are also formed by the hydrodynamical shocks due to the gas flow driven by the gravitational torques of the bar. Maciejewski et al. (2002) noticed that strong spiral shocks comparable to the principal shocks can propagate deep inside the IILR to make nuclear spiral when the sound speed of gas is high $\left(c_{s}=20 \mathrm{~km} \mathrm{~s}^{-1}\right)$, while for the low sound speed of gas $\left(c_{s}=5 \mathrm{~km} \mathrm{~s}^{-1}\right)$, the principal shocks can not penetrate the IILR barrier. Since our models except for the model M1 have no IILR, we expect that the trailing nuclear spiral patterns revealed in the models M2-M4 are formed by the spiral shocks that penetrate deep inside the ILR.

In order to see whether the nuclear spiral patterns shown in Fig. 4 are made of shocked gas particles, we examine the global distribution of gas particles which are shocked by supersonic flow, i.e., $-h \nabla \cdot \mathbf{v}>c_{s}$ (Englmaier \& Gerhard 1997), for the model M3 at the evolution time of $5 \tau_{\text {bar }}$ in Fig. 7. As is expected, the regions associated with the well known spiral shocks such as the principal shocks and 4/1-spiral shocks are well populated by these highly shocked gas particles. Thus, we believe that the nuclear spirals developed in the model M3 are formed by hydrodynamical shocks driven by the bar. However, there are some differences between the nuclear spiral pattern revealed by the distribution of all the gas particles in the central kiloparsec, as shown in Fig. 4, and that of the shocked gas particles in Fig. 7. The nuclear spiral shown in Fig. 4 displays a symmetric two-armed spiral pattern that winds up less than $\frac{3}{2} \pi$ in the azimuthal angle with similar density over the whole extension, whereas the spiral pattern made by shocked gas particles in Fig. 7 shows a somewhat sudden density drop near the azimuthal angle of $\sim \frac{3}{4} \pi$ but it extends upto $2 \pi$. There is a slight mismatch between the locations of these two spiral patterns. The loci of the local density maximum representing the nuclear spiral pattern in Fig. 4 are located just outside the nuclear spiral pattern revealed by shocked gas particles. But, this mismatch between the locations of the two spiral patterns is consistent with the general pattern of density distributions in the shocked regions. The loci of the highly shocked gas particles represent the shock front, while the regions of density maximum represented by the nuclear spiral pattern in Fig. 4 are located in the post-shock region. The density drop in the spiral pattern of shocked gas particles seems to be caused by the process of orbital switching from the x1-orbits to the x2-orbits.

Fig. 8 displays the distribution of shocked gas particles in the central kiloparsec of the models M1-M4. We selected earlier evolution time (i.e., at $3 \tau_{b a r}$ ) for the models M3 and M4, since the models with high sound speed evolve faster than the low sound speed models. As can be seen in Fig. 8, the distributions of shocked gas particles in the central kiloparsec of all the selected models reveal well defined spiral patterns which look very similar to the nuclear spiral shock pattern seen in Fig. 7. All the models show a smooth connection of nuclear spiral shocks with the principal shocks and a drop in the arm amplitude near the 
azimuthal angle of $\frac{3}{4} \pi$. But, for the models M1-M4, they extend upto winding angles of less than $\frac{3}{2} \pi, \sim \frac{3}{2} \pi, \sim 2 \pi$ and more than $2 \pi$, respectively. Thus, it seems apparent that the symmetric two-armed nuclear spirals can be formed by the bar-driven spiral shocks, regardless of the sound speed in the gas and the presence or absence of the central SMBH. However, the trailing nuclear spiral shocks can not be strong enough to maintain the nuclear spiral pattern in the later evolutions when regions of the principal shock are cleared of gas. Since the devoid of gas in regions of the principal shock is due to the lack of gas recycling in our models, we expect that the bar-driven spiral shocks can maintain the nuclear spiral arms even in the later evolutions if we supply the gas continuously. In case of the model M4 which shows the fastest evolution, only a small fraction of highly shocked particles can penetrate deep inside the ILR after $\sim 3 \tau_{\text {bar }}$ to make nuclear spiral arms. But, this weakening of the trailing nuclear spiral shocks occurs at $\sim 7 \tau_{\text {bar }}$ for the model M2, since it shows the slowest evolution. For the model M1 which has an IILR at $\sim 0.3 \mathrm{kpc}$, the trailing nuclear spiral shocks disappear after $\sim 10 \tau_{\text {bar }}$ due to the development of leading nuclear spiral shocks propagating outward from the IILR where the infalled gas particles are accumulated due to the barrier caused by this resonance and acquire angular momentum from the bar.

\subsection{Gas Inflow}

As shown in Fig. 2, the evolution of the gaseous disk leads to the redistribution of the gas particles. Although some fractions of the gas particles move outward by gaining angular momentum from the bar, a larger fractions of the gas particles move inward due to the loss of angular momentum. Since nuclear features are formed by the gas that moves inward across the ILR, it is important to understand the gas inflow in the central kiloparsec in detail. Moreover, the gas inflow deep inside the ILR may be closely related to the fueling mechanism of AGNs. Fig. 9 presents the time evolution of the fraction of particles accumulating inside two radial zones of $1.5 \mathrm{kpc}$ and $0.2 \mathrm{kpc}$. Since the ILR (the OILR for the model M1) is located near $1.5 \mathrm{kpc}$, the gas accumulated inside the radial zone of $1.5 \mathrm{kpc}$ shown in the left panel of Fig. 9 reflects the gas inflow across the ILR which is characterized by a large amount of gas inflow in the early phase of evolution with little difference among the models.

However, there are remarkable differences in the gas inflow close to the nucleus. As can be inferred from the time evolution of the fraction of gas particles accumulating inside the inner $0.2 \mathrm{kpc}$ of the modeled gaseous disks shown in the right panel of Fig. 9, the models M1 and M2 have negligible gas inflow into the central hundred parsecs except for some inflow around $\sim 10 \tau_{\text {bar }}$ in the model M1, while the models M3 and M4 show appreciable inflow throughout the evolution. Furthermore, it is also apparent that the gas inflow rate increases 
with the sound speed for the models with the central SMBH. As a result, the highest gas inflow rate is found for the model M4, which is a factor of $\sim 5$ larger than that of the model M3. The correlation between the amount of gas inflow close to the center and the gas sound speed is similar to that found by Patsis \& Athanassoula (2000) although their models do not take into account the central SMBHs. Apart from this consistency, it is also important to note that due to lack of resolution, the models considered by Patsis \& Athanassoula (2000), comparable to our model M1, do not develop a nuclear leading spiral. However, they show much larger inflow towards the center as sound speed increases.

The different amount of gas inflow among the models with the same potential but different sound speeds implies that angular momentum evolution depends on the sound speed in the gas, since the gas particles can move inward only if they lose their angular momentum. Fig. 10 shows the dependence of angular momentum evolution on the sound speed in the gas. The total angular momentum inside the bar radius (i.e., inside $r=3$ kpc) where the inflow of gas is taking place decreases fastest in the model M4 which has the highest sound speed in the gas (i.e., $c_{s}=20 \mathrm{~km} \mathrm{~s}^{-1}$ ), while it decreases slowest in the model M2 with $c_{s}=10 \mathrm{~km} \mathrm{~s}^{-1}$. Thus, it suggests for the models with the central SMBH that the gas particles in the high sound speed mediums lose their angular momentum more easily than those in the low sound speed mediums. The correlation between the rate of angular momentum loss and the sound speed in the gas can be understood if we consider the fact that the viscosity of the hot gas is greater than that of the cold gas (see Eq. (6)). Besides this dependence of angular momentum loss on the sound speed for the models having an $\mathrm{SMBH}$, it is also apparent that the presence of central SMBH much affects the evolution of total angular momentum inside the bar radius in the case of low sound speed medium (i.e., $c_{s}=10 \mathrm{~km} \mathrm{~s}^{-1}$ ), since it decreases faster in the model M1 than in the model M2. This suggests that the gas particles in the cold gas models can lose their angular momentum relatively easily for the model without an SMBH (i.e., the model M1) than that with an SMBH (i.e., the model M2).

There is some difference in the gas inflow between the models M1 and M2 that assume the same sound speed but different potentials due to the absence or the presence of an SMBH. The larger amount of gas inflow in the model M1 than in the model M2 around $\sim 10 \tau_{\text {bar }}$ is due to the larger $F_{T a n} / F_{R a d}$ in the central kiloparsec of the model M1, since the model M1 does not consider the central SMBH. The abrupt drop after $\sim 10 \tau_{\text {bar }}$ of the fraction of gas particles accumulated inside the central $0.2 \mathrm{kpc}$ of the model M1 is resulted from the onset of the outflow at the IILR which is caused by the change in the sense of bar torques at this resonance (Combes et al. 2002). Particles gain angular momentum at the IILR and can move outward from there. This outward gas flow leads to the formation of leading nuclear spiral in later times. 
It is interesting to see whether the gas inflow of our models can supply enough fuels for the AGNs. To do this, we estimated the mean inflow rates across the radius of $0.2 \mathrm{kpc}$ which is inside the IILR of the model M1. But as expected from Fig. 9, the mean inflow rate of the model M1 and M2 are negligibly small and can not fuel the AGNs. However, the mean inflow rates within the central $0.2 \mathrm{kpc}$ of the models M3 and M4 are $0.004 \mathrm{M}_{\odot} \mathrm{yr}^{-1}$ and $0.013 \mathrm{M}_{\odot} \mathrm{yr}^{-1}$, respectively. If we consider that the total mass of the gaseous disk is taken as $4 \times 10^{8} \mathrm{M}_{\odot}, \sim 1 \%$ and $\sim 3 \%$ of total gaseous disk mass are infalled close to the nucleus within $10^{9} \mathrm{yr}$ for the models M3 and M4, respectively. Since about half of this gas moves into the radius of $0.1 \mathrm{kpc}$, the nuclear spirals developed in the models M3 and M4 can provide fuels for the nearby AGNs.

\section{DISCUSSION}

\section{1. $\quad$ Effect of Sound Speed in the presence of SMBH}

The effect of the sound speed on the gas flow is somewhat well understood when there is no SMBH. Englmaier \& Gerhard (1997) have noticed that the gas flow in the low sound speed makes the off-axis principal shocks with a nuclear ring, while that in the high sound speed $\left(c_{s} \gtrsim 25 \mathrm{~km} \mathrm{~s}^{-1}\right)$ developes the on-axis ones with no gas on the $x_{2}$-orbits and hence, this represents the continuous inflow towards the center. A similar tendency for the principal shocks to move towards the bar major axis as sound speed increases has been found by Patsis \& Athanassoula (2000). Besides these features, they also found that the gas inflow inside the radial zone near to the central regions increases with the sound speed.

Here we obtained similar results for the effect of the sound speed on the gas flow, including the shock locations and the inflow of gas, using mass models with and without central SMBHs. However, owing to better resolutions of our simulations, we can analyze the effect of the sound speed on the gas flows in the central kiloparsecs of galaxies where nuclear spirals are found to be present. There seems to be a clear distinction in the morphology of nuclear spirals formed in the central kiloparsecs according to the presence and absence of the central SMBHs. In the quasi-steady state, the nuclear regions of the modeled gaseous disks evolve to the trailing nuclear spirals whose winding angles depends on the sound speed in the gas when there is a central SMBH, whereas the lower sound speed model that has no SMBH evolves to a leading nuclear spiral. However, it should be noted that the formation of a leading nuclear spiral such as that of the model M1 is limited to some special cases. Even in the models with the same mass model as that of the model M1 but with different sound speeds, we do not find leading nuclear spirals similar to that found in the model M1. The models with higher sound speed $\left(c_{s}>15 \mathrm{~km} \mathrm{~s}^{-1}\right)$ show a development of elongated rings, 
while lower sound speed model $\left(c_{s}=5 \mathrm{~km} \mathrm{~s}^{-1}\right)$ display a double ring structure of which the thick outer ring is more round and aligned perpendicular to the bar and the thin inner ring that is located at the IILR is aligned to the bar.

A necessary condition for the formation of a leading nuclear spiral is the existence of an IILR but there are other conditions that are required for the the formation of a leading nuclear spiral. A favorable condition for the leading nuclear spiral is the lack of a large amount of gas inflow across the OILR because leading nuclear spiral shocks can not propagate outward when there are strong trailing nuclear spiral shocks from the OILR. In general, the leading spiral shocks are generated at the IILR due to the negative torques of the bar (Combes et al. 2002), while the trailing spiral shocks at the OILR are made by the orbital switching from the $x_{1}$-orbits to the $x_{2}$-orbits. In our model M1, the regions around the OILR are devoid of gas particles after $\sim 5 \tau_{\text {bar }}$ which weakens the trailing nuclear spiral shocks greatly. But, there is enough gas near the IILR to make strong leading nuclear spiral shocks, since gas particles are accumulated at the IILR due to the barrier caused by this resonance. Since the weakening of the trailing nuclear spiral shocks in our models is due to the lack of gas recycling in our simulations, we expect that strong trailing nuclear spiral shocks are more common features than the weak ones in realistic physical conditions. This means that the leading nuclear spiral is mostly a transient feature, if any, that can be formed in some specific physical conditions. It is the reason why the leading nuclear spirals are so rare in real galaxies.

The correlation between the winding angle of the trailing nuclear spirals and the gas sound speed in the presence of an SMBH that removes the IILR can be understood if we consider the dependence of the angular momentum evolution on the sound speed in the gas (see Fig. 10). The large winding angles of nuclear spirals in the higher sound speed models are due to the large amount of angular momentum loss which makes physical condition plausible for the innermost parts of nuclear spirals to reach very close to the center. On the other hand, the gas particles in the lower sound speed models lose their angular momentum slowly so that they can not spiral in close to the center. This interpretation is also supported by the correlation between the gas inflow in the very vicinity of the nucleus $(r \sim 0.2 \mathrm{kpc})$ and the sound speed in the gas (see Fig. 9). The quasi-steady state morphology of the trailing nuclear spirals are consistent with the prediction of the linear density wave theory in the sense that the spiral arms open out as sound speed in the gas increases (Englmaier \& Shlosman 2000). 


\subsection{Hydrodynamic Shock as an Origin of Nuclear Spirals}

The gas density waves proposed by Englmaier \& Shlosman (2000) is one of promising mechanism that can explain the formation of the grand-design nuclear spirals. But, it can be applicable only to the low amplitude spirals where no shocks exist because gas density waves can not propagate in the regions where shocks are strong enough to drive non-linear response of gas flows. There are several examples of grand-design nuclear spirals which have densities high enough for active star formation. The Hubble Space Telescope NICMOS $H$-band images of NGC 5427 and NGC 5614 clearly show young stellar populations in their nuclear spirals (Martini et al. 2003). Since the densities of their nuclear spirals are too high to be considered as linear, they can not be explained by the gas density waves of Englmaier \& Shlosman (2000) but by the hydrodynamic spiral shocks presented in this study. As shown in Fig. 7, the spiral shocks can penetrate deep inside the ILR, at least in early phase of evolutions ( $\mathrm{t} \lesssim 5 \tau_{\text {bar }}$ ) to wind upto $2 \pi$ radian in azimuthal angle although there is a certain drop of particle density at $\sim \frac{3}{4} \pi$. The shock nature of the nuclear spiral is also addressed by Maciejewski et al. (2002) from the grid-based hydrodynamical simulations. In their high sound speed run $\left(c_{s}=20 \mathrm{~km} \mathrm{~s}^{-1}\right)$, they found that a two-armed nuclear spiral is a direct continuation of the principal shock along the leading edge of the bar and it reaches close to the nucleus well beyond the IILR where the gas density waves can not penetrate. In their

model, the strength of shocks, as measured by $\operatorname{div}^{2} \mathbf{v}$, and the arm/interarm density contrast are comparable to those of the principal shocks, which indicates that such a nuclear spiral is well beyond the linear regime that can be explored in the density wave theory.

However, at the late phase of evolution when the gas flows become a quasi-steady state, we do not expect strong shocks deep inside the ILR (or the OILR when there are two ILRs) due to less orbital crossings, since most of the particles are already in the x2-orbits. Thus, the maintenance of nuclear spiral in the quasi-steady state is not made by the bar-driven spiral shocks but by the gas density waves proposed by Englmaier \& Shlosman (2000). But, since the weakening of the nuclear spiral shocks is due to the devoid of gas in regions of the principal shock, the nuclear spiral turns into density wave only when the driver of the nuclear spiral shocks ceases. Of course it is also possible that grand-design nuclear spirals can be made by gas density waves. But, nuclear spirals formed by gas density waves should be of low-amplitude so that the gas response could be considered as a linear wave (Englmaier \& Shlosman 2000). It is worth to note that gas density wave itself is triggered by the spiral shocks driven by the bar (Englmaier \& Shlosman 2000).

The self-gravity of gas is known to be effective to drive gas inflow by generating torques among gas clumps (Wada \& Habe 1992; Elmegreen 1994; Fukuda et al. 1998, 2000). However, the density of the gaseous disks should be high enough to make clumps by the gravitational 
instability. To clarify whether the inflow of the gas towards the center is due to the hydrodynamic shocks or due to the torques generated by the gas clumps, we calculated Toomre's $Q$-value in the several high density regions where the spiral arms are developed. If the value of the $Q \lesssim 1$, the gaseous disk would be gravitationally unstable, whereas $Q>1$ represents the stable gaseous disk. Toomre's $Q$-value of our models is expressed as

$$
Q \sim 3.44 \times 10^{-3} \frac{\kappa}{\sigma}\left(\frac{c_{s}}{10 k m s^{-1}}\right),
$$

where $c_{s}$ is the sound speed corresponding to each model, $\sigma$ is the surface density of the selected high density regions of the gaseous disk at a particular evolution time and $\kappa$ is the epicycle frequency at the location of each selected region. Here the units of the $\sigma$ and $\kappa$ are $2 \times 10^{5} \mathrm{M}_{\odot} \mathrm{pc}^{-2}$ and $929.5 \mathrm{~km} \mathrm{~s}^{-1} \mathrm{kpc}^{-1}$, respectively. We found that the surface density $\sigma$ of all the selected regions are found to be smaller than the term $3.44 \times 10^{-3} \kappa\left(c_{s} / 10 \mathrm{kms}^{-1}\right)$ of Eq. (17) and as a result, Toomre's $Q$-values are greater than unity (i.e., $Q>1$ ) for our models. The reason for the low surface density even at the densest regions of the gaseous disk is due to the small mass fraction of the initial gaseous disk, i.e., $M_{\text {gas }} / M_{G}=0.01$. Although some clumped structures seem to appear at later phase of evolutions for the model M4, it is clear that the self-gravity of the gas is not large enough to drive the gas particles inward. Thus, we confirm that the inflow of the gas particles towards the center to form the symmetric two-armed nuclear spiral is due to the hydrodynamic shocks induced by the gravitational torque of the bar rather than the torques among the gaseous clumps. Despite this fact, the gas self-gravity still takes its importance to include in the simulations, as it can affect the nuclear gaseous structure in the late phase of evolutions that we have already noticed in the case of models M1 and M4.

\section{CONCLUSIONS}

We have used the SPH technique to simulate the response of the gaseous disk to the moderately strong bar potentials in order to understand the formation mechanism of the symmetric two-armed nuclear spirals. We found that the gas flows driven by the gravitational torques of the bar lead to the formation of nuclear spirals whose morphology depends much on the sound speed of gas. Although SMBHs play a critical role in shaping the gravitational potential in the central kiloparsec to weaken or to remove the IILR that prevents the gas inflow close to the nucleus, the sound speed of gas seems to be the primary physical parameter which controls the gas flows deep inside the ILR to make nuclear features. In the quasi-steady state, the global morphological features of the modeled gaseous disks are not much affected by the sound speed in the gas although models with high sound speeds evolve faster than those with lower sound speeds. Since mass inflow from the disk is an essential process to 
make nuclear features, proper treatment of the global evolution of the gaseous disk is required to understand the nuclear features such as nuclear spirals.

The symmetric two-armed nuclear spirals are formed by the hydrodynamic spiral shocks induced by the gravitational torque of the bar when there is a central SMBH. The masses of SMBHs are assumed to be about $1 \%$ of the total visible mass of the model galaxies that is massive enough to remove the IILR. The symmetric two-armed nuclear spirals whose innermost parts reach close to the center of galaxies are likely to form in the high sound speed medium $\left(c_{s} \gtrsim 15 \mathrm{~km} \mathrm{~s}^{-1}\right)$, while the ring-like nuclear spirals develop in the low sound speed medium. In some special physical conditions like those assumed in the cold gas models of present study, a leading nuclear spiral can be developed between the IILR and the OILR when there is no central SMBH. In this case, there is little inflow of gas inside the IILR due to IILR barrier. In the presence of a central SMBH, the mean gas inflow rate inside the central hundred parsecs increases with the sound speed. For the model that assumes sound speed of $c_{s}=20 \mathrm{~km} \mathrm{~s}^{-1}$, the mean gas inflow rate within $0.1 \mathrm{kpc}$ is about $\sim 0.007 \mathrm{M}_{\odot} \mathrm{yr}^{-1}$, which suggests that $\sim 1.75 \%$ of the total gaseous disk mass moves into the radius of $0.1 \mathrm{kpc}$ within $10^{9} \mathrm{yr}$. Thus, the nuclear spirals formed in the hot interstellar medium seem to be effective for fueling the nearby AGNs. Finally, the trailing nuclear spiral shocks are too weak to support the nuclear spiral in the later phase of evolution when there is little gas inflow across the ILR. Thus, nuclear spiral turns into density wave when the driver of spiral shocks ceases and the spiral arms open out as sound speed in the gas increases.

We thank the anonymous referee for useful remarks and suggestions which improve the present paper enormously. We also wish to express our sincere thanks to Dr. Roger Fux who provides the PMSPH code. HBA thanks Prof. Hyesung Kang for valuable discussion and comments on the numerical simulations. PT would like to express his sincere thanks to ARCSEC (Astrophysical Research Center for the Structure and Evolution of the Cosmos) for providing support through the postdoctoral fellowship which made this study possible. This work is supported in part by grant No. R01-1999-00023 from the Korea Science Engineering Foundation (KOSEF). Most of the computations are conducted using the super computer facilities in the Korea Institute of Science and Technology Information (KISTI). 


\section{REFERENCES}

Ann, H.B. 2001, AJ, 121, 2515

Ann, H.B., \& Lee, H.M. 2000, JKAS, 33, 1

Ann, H.B., \& Lee, H.M. 2004, ApJL, submitted (astro-ph/0408280)

Athanassoula, E. 1992, MNRAS, 259, 345

Balsara, D.S. 1995, J. Comput. Phys., 121, 357

Benz, W. 1990, in The Numerical Modelling of Nonlinear Stellar Pulsations, ed. J.R. Buchler, NATO AST Ser. C 302, 269

Binney, J., \& Tremaine, S. 1987, in Galactic Dynamics, 42

Combes, F., Boisse, P., Mazure, A., \& Blanchard, A. 2002 Galaxies and Cosmology (New York: Springer), 172

Contopoulos, G., \& Papayannopoulos, T. 1980, A\&A, 92, 33

Elmegreen, B.G 1994, ApJ, 425, L73

Elmegreen, B.G., Elmegreen, D. M., Brinks, E., Yuan, C., Kaufman, M., Klaric, M., Montenegro, L.; Struck, C., \& Thomasson, M. 1998, ApJ, 503, L119

Englmaier, P., \& Gerhard, O.E. 1997, MNRAS, 287, 57

Englmaier, P., \& Shlosman, R.S. 2000, ApJ, 528, 677

Freeman, K.C. 1966, MNRAS, 134, 1

Freeman, K.C. 1970, ApJ, 160, 811

Fukuda, H., Wada, K., \& Habe, A. 1998, MNRAS, 295, 463

Fukuda, H., Habe, A., \& Wada, K. 2000, ApJ, 529, 109

Fux, R. 1999, A\&A, 345, 787

Fux, R. 2001, JKAS, 34, 255

Haro, G. 1956, AJ, 61, 178

Hasan, H., Pfenniger, D., \& Norman, C. 1993, ApJ, 409, 91 
Heller, C., \& Shlosman, I. 1994, ApJ, 424, 84

Heller, C., Shlosman, I., \& Englmaier, P. 2001, ApJ, 553, 661

Hut, P., Makino, J., \& McMillan, S. 1995, ApJ, 443, L93

Knapen, J.H., Beckman, J.E., Heller, C.H., Shlosman, I., \& de Jong, R.S. 1995, ApJ, 454, 623

Laine, S., Knapen, J.H., Perez-Ramiez, D., Doyon, R., \& Nadeau, D. 1999, MNRAS, 302, L33

Laine, S., Knapen, J.H., Perez-Ramiez, D., Englmaier, P., \& Matthias, M. 2001, MNRAS, 324,891

Long, K., \& Murali, C. 1992, ApJ, 397, 44

Maciejewski, W., Teuben, P.J., Sparke, L.S., \& Stone, J.M. 2002, MNRAS, 329, 502

Maciejewski, W. 2003, in EAS Publications Series, Volume 10, the proceedings of the JENAM 2002, Galactic and Stellar Dynamics, ed. C. Boily, P. Patsis, C. Theis, S. Portegies Zwart, \& R. Spurzem (EDP Sciences), 3 (astro-ph/0302250)

Marconi, A., \& Hunt, L.K. 2003, ApJ, 589, L21

Martini, P.,\& Pogge R.W. 1999, AJ, 118, 2646

Martini, P., Regan, M.W., Mulchaey, J.S., \& Pogge, R.W. 2003, ApJ, 146, 353

Monaghan, J. 1992, ARA\&A, 30, 543

Montenegro, L. E., Yuan, C., \& Elmegreen, B. G. 1999, ApJ, 520, 592

Patsis, P.A., \& Athanassoula, E. 2000, A\&A, 358, 45

Pfenniger, D., \& Friedli, D. 1993, A\&A, 270, 561

Phillips A.C., Illingworth, G.D., MacKenty, J.W., \& Franx, M. 1996, AJ, 111, 1566

Pogge R.W., \& Martini, P. 2002, ApJ, 569, 624

Regan M.W., \& Mulchaey J.S., 1999, AJ, 117, 2676

Sersic, J. L. 1956, Obs, 78, 123

van der Kruit, P.C., \& Shostak, G.S., 1984, A\&A, 134, 258 
Wada, K. 1994, PASJ, 46, 165

Wada, K., \& Habe, A. 1992, MNRAS, 258, 82

Wada, K., \& Koda, J. 2004, MNRAS, 349, 270 

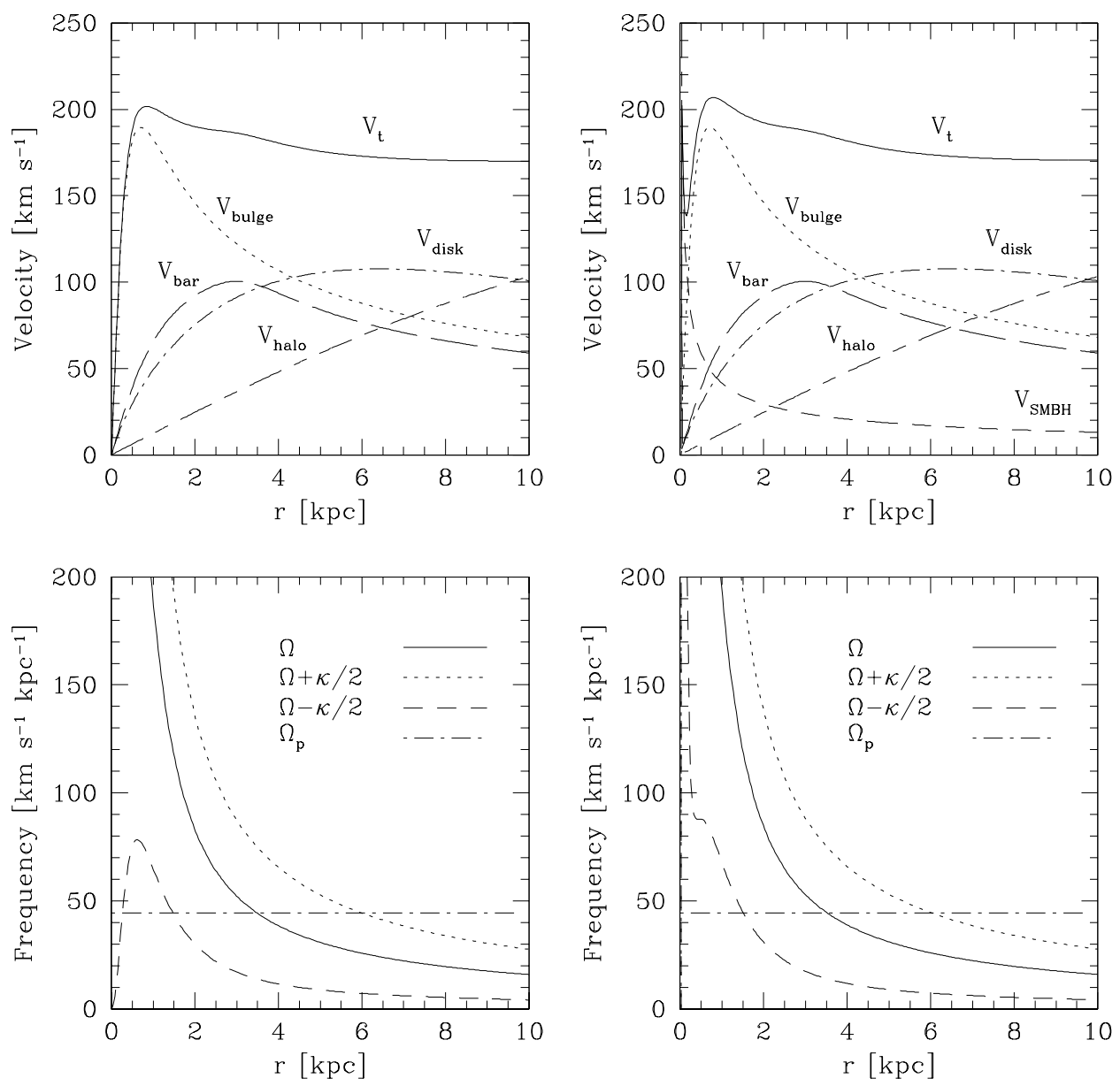

Fig. 1.- Rotation curves and angular frequencies of model galaxies. Because of nonaxisymmetric nature of the bar, its contribution is included here after averaging it axisymmetrically. The left panels are for the model M1 that has no SMBH, whereas the right panels are for the model M2 with SMBH whose mass is about $1 \%$ of the total visible mass of galaxy. The horizontal dot-dash lines in the bottom panels represent the bar pattern speed of $\Omega_{p}=44.6 \mathrm{~km} \mathrm{~s}^{-1} \mathrm{kpc}^{-1}$. 


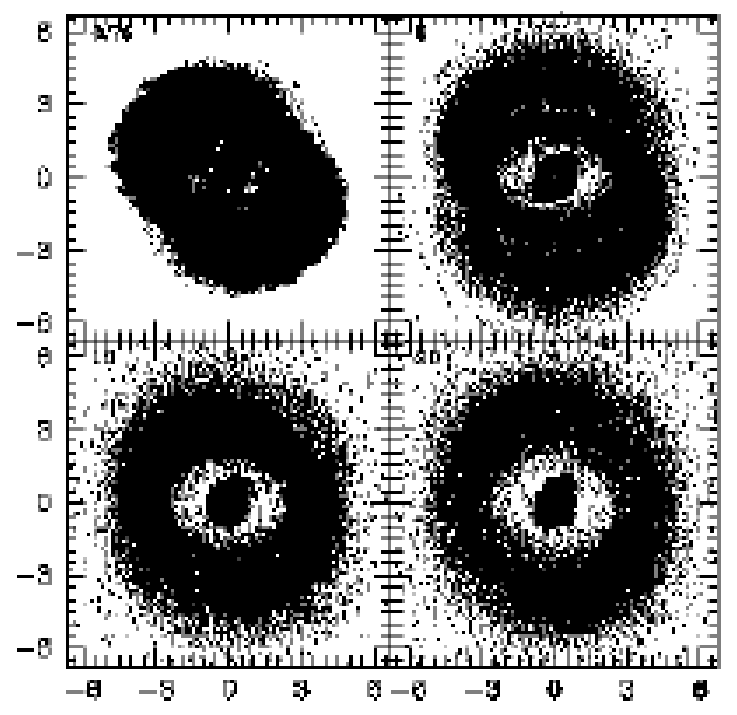

Fig. 2.- Global evolution of gaseous disk for the model M3. The number in the top left corner of each panel is the evolution time in the unit of $\tau_{\text {bar }} \sim 1.4 \times 10^{8} \mathrm{yr}$. The bar lies horizontally and the box size is $13.4 \mathrm{kpc}$ in one dimension. 


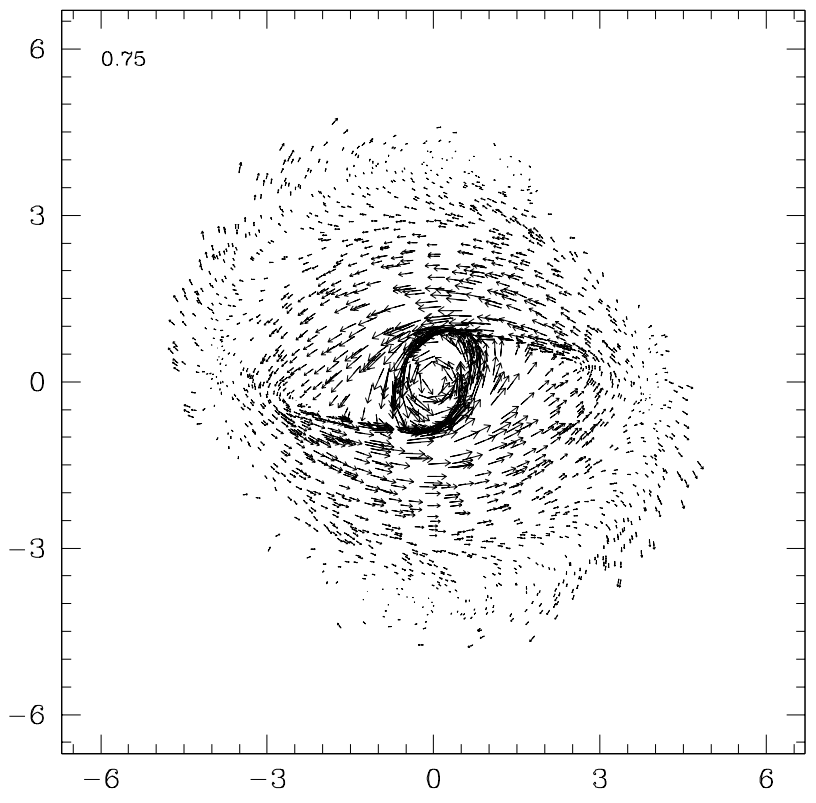

Fig. 3.- Velocity field of the Model M3 at evolution time of $0.75 \tau_{\text {bar }}$. The velocity of each particle is represented by the arrow whose length is proportional to the speed. To achieve better resolution, the velocities of randomly selected $2 \times 10^{3}$ gas particles are plotted. The bar lies horizontally and the box size is $13.4 \mathrm{kpc}$ in one dimension. 


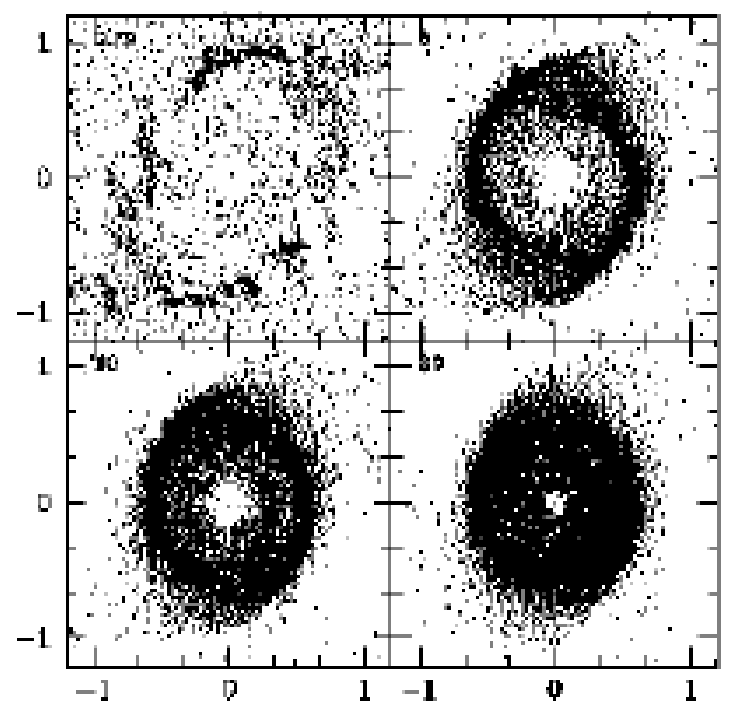

Fig. 4.- Evolution of the nuclear regions of the gaseous disk for the model M3. The number in the top left corner of each panel is the evolution time in the unit of $\tau_{\text {bar }}$. The bar lies horizontally and the box size is $2.4 \mathrm{kpc}$ in one dimension. 


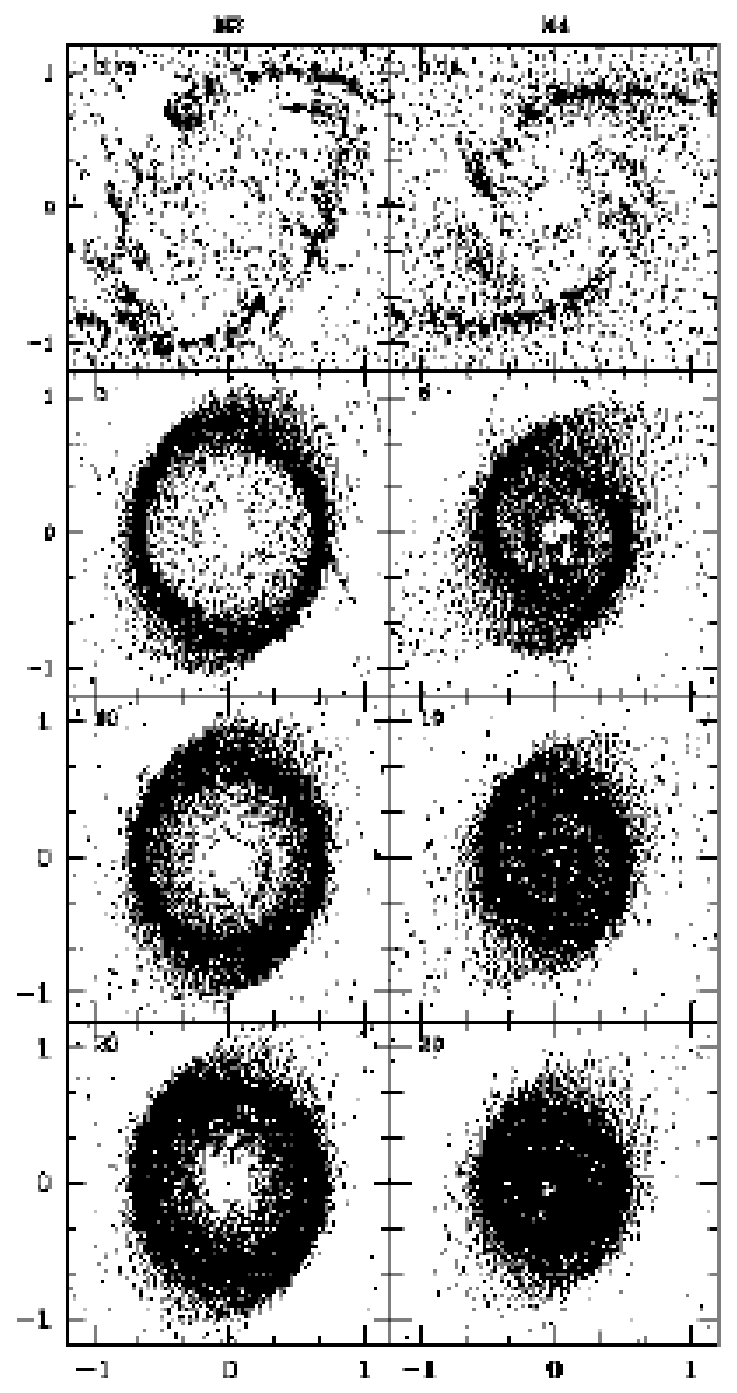

Fig. 5.- Same as Fig. 4, but for the models M2 and M4. The left panels are for the model M2, whereas the right panels are for the model M4. 


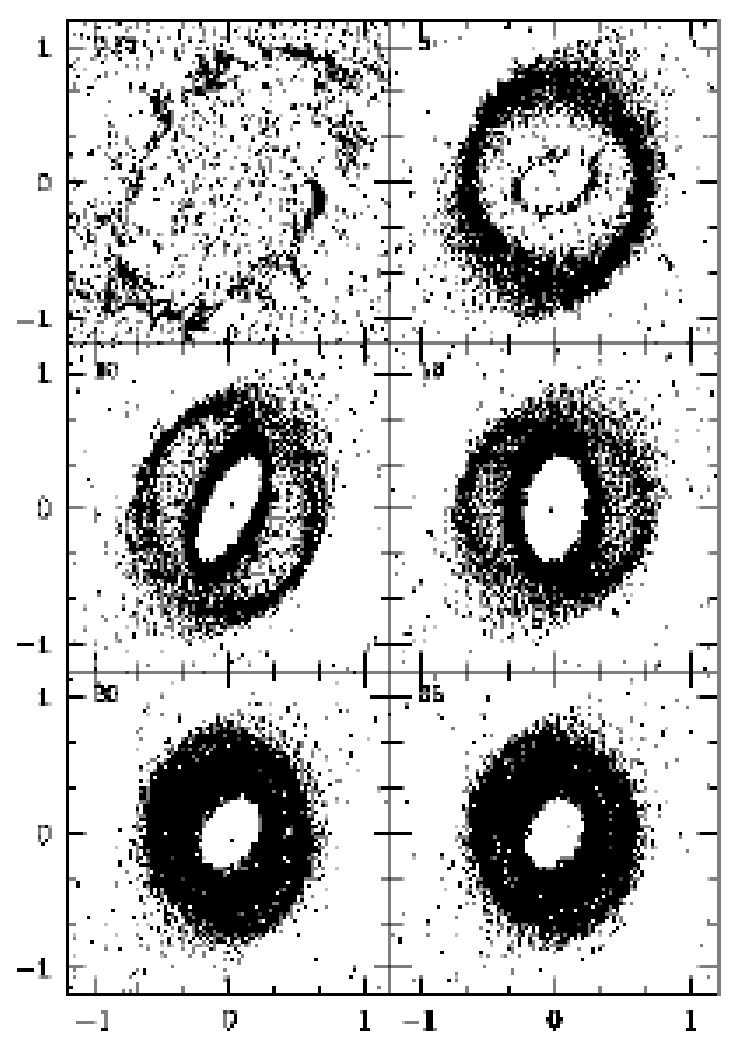

Fig. 6.- Same as Fig. 4, but for the model M1. 


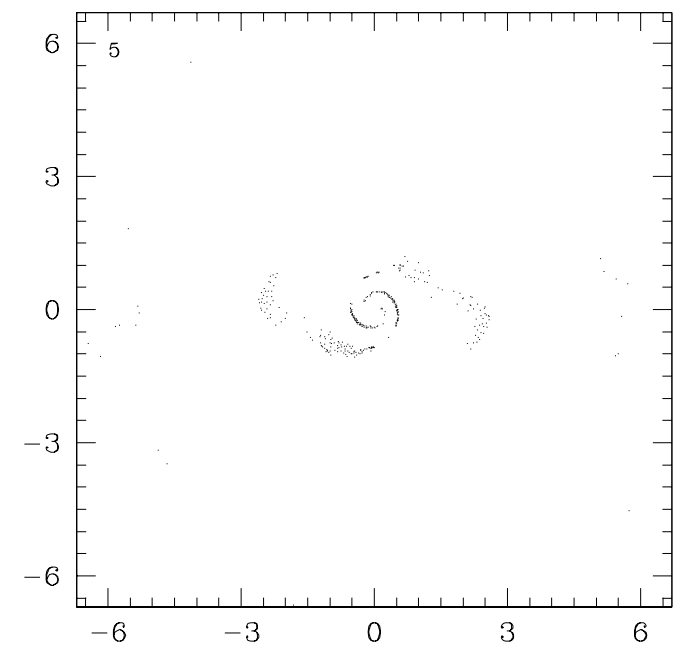

Fig. 7.- Distribution of the gas particles that are shocked by supersonic flow (i.e., $-h \nabla \cdot \mathbf{v}>$ $c_{s}$ ) for the model M3 at evolution time of $5 \tau_{\text {bar }}$. The bar lies horizontally and the box size is $13.4 \mathrm{kpc}$ in one dimension. 


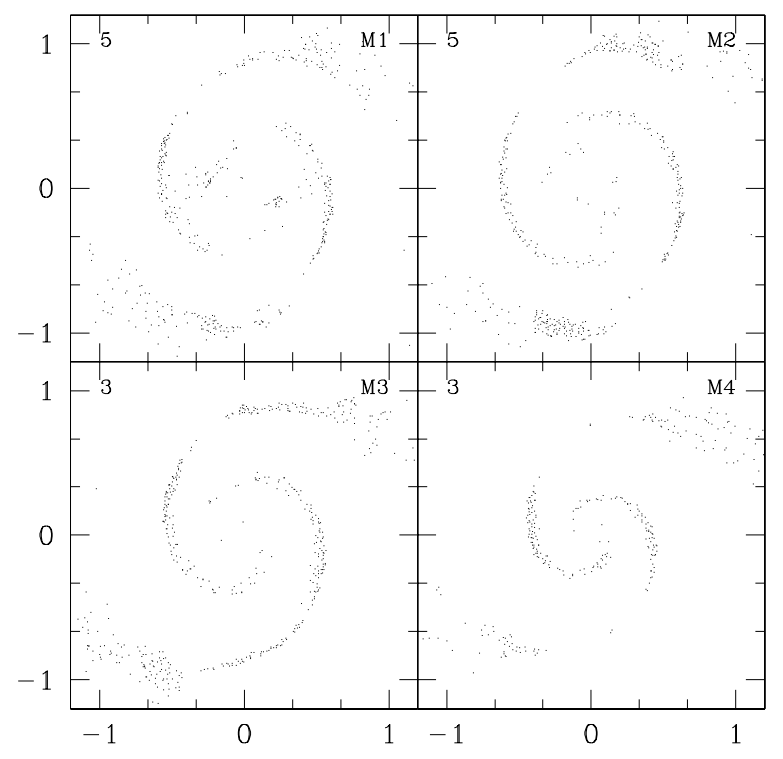

Fig. 8.- Nuclear distribution of the gas particles that are shocked by supersonic flow (i.e., $-h \nabla \cdot \mathbf{v}>c_{s}$ ) for the models M1-M4. The models are indicated in the top right corner and the evolution time in the unit of $\tau_{b a r}$ is given in the top left corner of each panel. The bar lies horizontally and the box size is $2.4 \mathrm{kpc}$ in one dimension. 

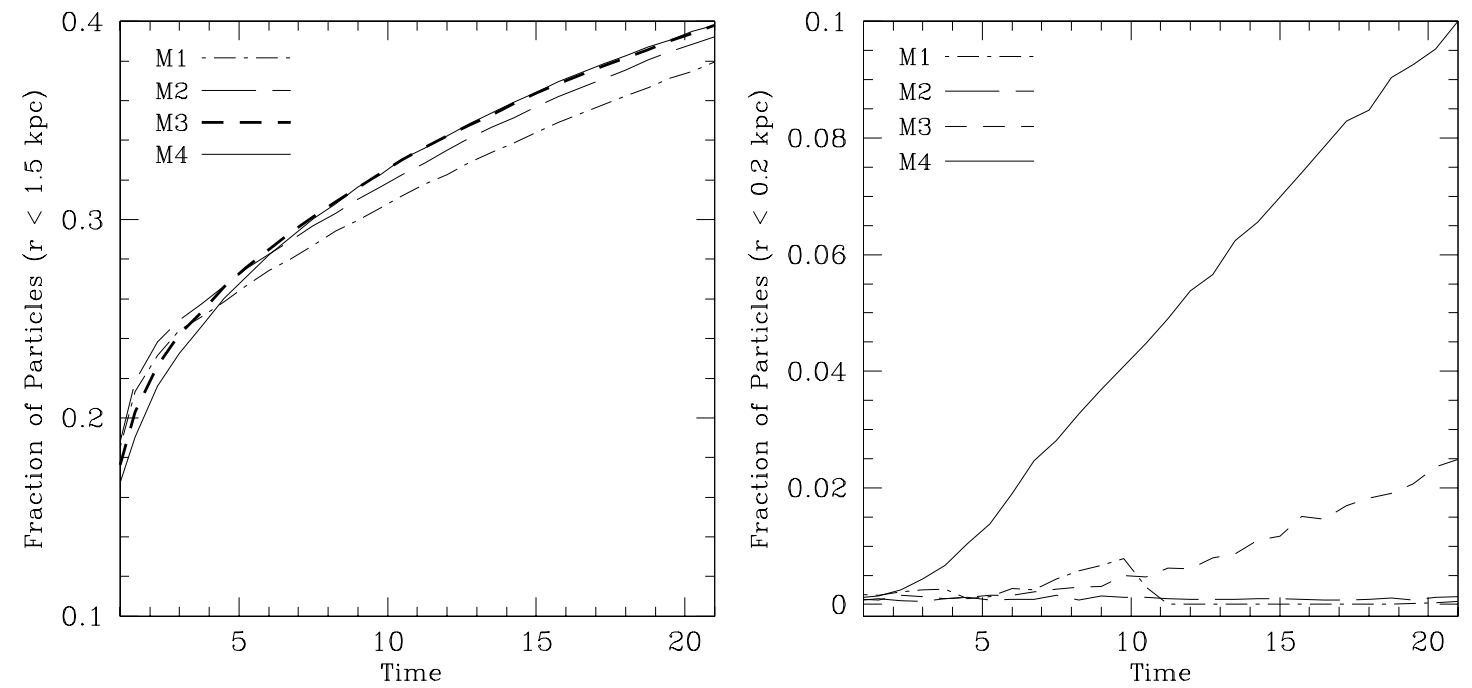

Fig. 9.- The inflow of the gas particle in the models M1-M4 for two different radial zones. The time evolution of the fraction of particles accumulated inside the radius of $1.5 \mathrm{kpc}$ is presented in the left panel, while that for the radius of $0.2 \mathrm{kpc}$ is shown in the right panel. The time is given in the unit of $\tau_{\text {bar }}$. The line styles corresponding to each model are designated in the top left corner of each panel. 


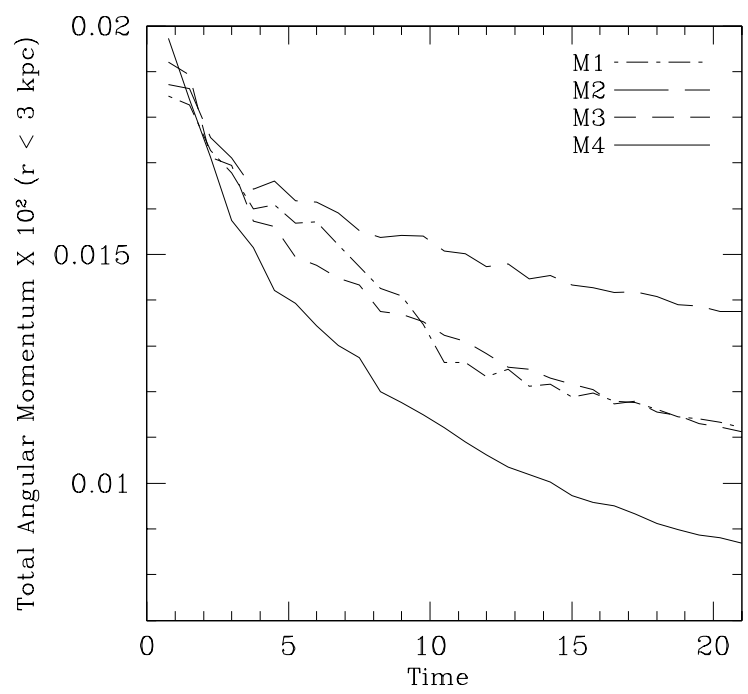

Fig. 10. - The time evolution of the total angular momentum of the gas particles accumulated within the radius of $3 \mathrm{kpc}$ (i.e., the bar radius) for the models M1-M4. The evolution time is given in the unit of $\tau_{\text {bar }}$. The line styles corresponding to each model are given in the top right corner of the panel. 